Instituto Internacional de Investigación y Desarrollo Tecnológico Educativo INDTEC, C.A.

DOI: https://doi.org/10.29394/Scientific.issn.2542-2987.2020.5.E.9.185-204

OAI-PMH: http://www.indteca.com/ojs/index.php/Revista Scientific/oai

Artículo Original / Original Article

\title{
Evaluación de riesgos psicosociales en servidores policiales especializados
}

Autores: Nathalie Soledad López Arroyo Universidad Internacional SEK, UISEK nslopez.mpp@uisek.edu.ec

Quito, Ecuador https://orcid.org/0000-0002-5928-1930

Erika Elizabeth Torres Rueda Universidad Internacional SEK, UISEK eetorres.mpp@uisek.edu.ec

Quito, Ecuador https://orcid.org/0000-0001-6006-8689

Carmen Eugenia Mora Mora Universidad Internacional SEK, UISEK cemora.mpp@uisek.edu.ec

Quito, Ecuador https://orcid.org/0000-0002-5252-2308

Esteban Ricaurte Ricaurte Universidad Internacional SEK, UISEK esteban.ricaurte@uisek.edu.ec

Quito, Ecuador https://orcid.org/0000-0001-8702-7449

\section{Resumen}

Esta investigación tiene como objetivo identificar el riesgo psicosocial y burnout en el personal directivo y técnico operativo de la Dirección Nacional de Delitos Contra la Vida, Muertes Violentas, Desapariciones, Extorsión y Secuestro de la Policía Nacional (DINASED), determinando el impacto tanto a nivel físico como emocional por encontrarse en contacto permanente con casos de desaparición de personas, muertes violentas, tentativas de asesinato y secuestro. La presente investigación se realizó con una metodología cuantitativa, de tipo experimental y con un corte trasversal. Se llevó a cabo con una población de 170 servidores policiales. El análisis se realiza a través de la Aplicación de Reactivos Psicométricos tales el cuestionario de riesgos psicosociales 3.1 y el Cuestionario de Maslach Burnout Inventory, herramientas que fueron seleccionadas debido a su fundamento científico, diseño, fiabilidad y validez. Los resultados muestran un factor de riesgo significativo relacionado con: tiempo de trabajo, demanda psicológica, participación-supervisión y carga de trabajo. Se evidencia elevado cansancio laboral o burnout, cansancio emocional y despersonalización, debidos al tipo de funciones y al tiempo que llevan realizándolas. Concluimos que si existe un riesgo psicosocial y burnout significativo.

Palabras clave: evaluación; psicoterapia; policía.

Cómo citar este artículo:

López, N., Torres, E.., Mora, C., \& Ricaurte, E. (2020). Evaluación de riesgos psicosociales en servidores policiales especializados. Revista Scientific, 5(Ed. Esp.), 185-204, e-ISSN: 2542-2987. Recuperado de: https://doi.org/10.29394/Scientific.issn.2542-2987.2020.5.E.9.185-204

Fecha de Recepción: 10-04-2020
Fecha de Aceptación: 20-06-2020
Fecha de Publicación: 05-08-2020 
OAI-PMH: http://www.indteca.com/ojs/index.php/Revista_Scientific/oai

Artículo Original / Original Article

\title{
Psychosocial risk assessment in specialized police servers
}

\begin{abstract}
This research aims to identify the psychosocial risk and burnout in the management and technical personnel of DINASED, determining the impact both physically and emotionally due to their vulnerability to risk factors for being in permanent contact with people exposed to suffering due to disappearances of relatives, violent deaths, attempted murder and kidnapping. The present investigation is carried out with a population of 170 police officers divided into two groups by operational and administrative function. The analysis is carried out through the Application of Psychometric Reagents such as the 3.1 psychosocial risk questionnaire and the Maslach Burnout Inventory Questionnaire, tools that were selected due to their scientific basis, design, reliability and validity. The results show a significant risk factor that prevails in factors such as: work time, psychological demand, participation / supervision and workload. High work fatigue or burnout -especially in the unit of violent deaths- is due to emotional fatigue and depersonalization phenomena, resulting of the kind of functions and time they perform them.
\end{abstract}

Keywords: evaluation; psychotherapy; police.

How to cite this article:

López, N., Torres, E., Mora, C., \& Ricaurte, E. (2020). Psychosocial risk assessment in specialized police servers. Revista Scientific, 5(Ed. Esp.), 185-204, e-ISSN: 2542-2987. Recovered from: https://doi.org/10.29394/Scientific.issn.2542-2987.2020.5.E.9.185-204

Date Received: 10-04-2020
Date Acceptance:

20-06-2020
Date Publication:

05-08-2020 


\section{Introducción}

El agotamiento laboral o síndrome de burnout, para Maslach (2009): es un conjunto de signos y síntomas psicológicos, que afrontan los trabajadores al estar sometidos a duras condiciones de estrés en su ámbito laboral. Parafraseando a Moreno (2011): estas condiciones intensifican los riesgos psicosociales, comprendiendo como aquellos factores que impactan en la salud física y/o emocional de los trabajadores que presentan características propias que hacen más difícil su manejo, evaluación y control.

En el ámbito laboral, Álvarez (2006): expone que los factores de riesgo son todas las condiciones presentes en una situación laboral los mismos que se encuentran relacionados con la organización o estructura, contenido del trabajo, ejecución de tareas, afectando el equilibrio o salud (física, psicológica y social) del colaborador como al desarrollo del trabajo.

Dichas condiciones serán disímiles en cada uno de los trabajadores, ya que su reacción dependerá de sus habilidades de afrontamiento que varían en cuanto a sus funciones a desempeñar y de componentes tales como costumbres, creencias, valores e interacción en su medio laboral la misma que influye en muchos casos a la estabilidad psíquica o emocional.

El tratamiento diario con los ciudadanos que presentan problemas y que muchas veces no siempre resulta eficaz, puede transformarse en una importante causa de estrés negativo para los servidores policiales, conduciéndolo a sentimientos de culpa, despersonalización que es una consecuencia que está relacionada con el burnout.

En el Ecuador, el Ministerio del Trabajo (MDT, 2017): en su Acuerdo Ministerial señala que:

En todas las empresas e instituciones públicas y privadas, que cuenten con más de 10 trabajadores, se deberá implementar el programa de prevención de riesgos psicosociales, en base a los parámetros y formatos establecidos por la Autoridad Laboral, mismo que deberá contener acciones para fomentar 
una cultura de no discriminación y de igualdad de oportunidades en el ámbito laboral (art. 9).

En nuestro país muy poco se ha estudiado sobre el estrés laboral y el agotamiento de los servidores policiales, ya que la sociedad se ha enfocado en criticar, cuestionar y juzgar su desempeño laboral, su comportamiento y la relación que mantiene con la ciudadanía al momento de acudir a una emergencia, maximizando a través de los medios de comunicación dicho cuestionamiento.

Siendo este el panorama general, consideramos que el personal directivo y técnico operativo de la Dirección Nacional de Delitos Contra la Vida, Muertes Violentas, Desapariciones, Extorsión y Secuestro de la Policía Nacional (DINASED) de la Policía Nacional, es particularmente vulnerable a factores de riesgo por encontrarse en contacto permanente con personas expuestas al sufrimiento por desapariciones de familiares, muertes violentas, tentativas de asesinato y secuestros.

El grupo de servidores policiales en estudio, se encuentran constreñidos a intervenir bajo condiciones adversas hasta la culminación de sus procedimientos. En muchas ocasiones, ellos mismos son objeto de amenazas dentro de los procesos investigativos, se exponen al cuestionamiento permanente de los procedimientos que llevan a cabo, no cuentan en todos los casos con el respaldo de un equipo de trabajo dinámico, participativo y con el conocimiento al mismo nivel, entre otros factores. A esto se añade, que se exponen a una sobrecarga laboral, con horarios extendidos, fuera de su entorno familiar, sometidos a constantes rotaciones y traslados laborales dentro de entornos y condiciones físicas inadecuadas.

La disposición de sus condiciones de trabajo se encuentra precarizadas, ya que conllevan inseguridad, incertidumbre y falta de garantías añadiendo que no cuentan con medios logísticos, insumos básicos, teniendo 
que utilizar medios económicos propios para responder al límite de tiempo asignado por cada caso que se encuentre bajo su responsabilidad.

Por otro lado, la influencia de medios de comunicación y redes sociales dentro de su mecanismo de producción, circulación y reproducción de discursos condiciona la creación de sentido en torno a la temática, interfiriendo en las vías formales de denuncia, convirtiendo a un elevado número de casos en "casos de connotación social o presidenciales", debido a su exposición mediática, esta situación dificulta particularmente los procesos investigativos.

En este contexto, la alteración de la esfera afectiva en los agentes investigativos, exacerba los niveles de estrés al que están expuestos en el marco del cumplimiento de sus actividades y procedimientos policiales. La DINASED desde su creación y hasta el momento, no cuenta con un estudio que permita identificar los riesgos psicosociales y el nivel de burnout en este grupo de servidores policiales que cumplen funciones como agentes investigativos. Por lo tanto, es trascendental identificar: ¿Cuáles son los factores de riesgo psicosocial y los niveles de burnout en el personal directivo y técnico operativo de la DINASED de la ciudad de Quito, Ecuador?

Esta investigación es relevante para la Psicología debido a que el personal de estudio brinda un servicio de atención directa a usuarios, familiares y víctimas de desapariciones, muertes violentas, secuestros y tentativas de asesinato. Siendo importante la estabilidad y equilibrio emocional del personal directivo y técnico que labora en esta entidad policial, además de que sería una forma eficaz de incrementar la calidad de los procesos humanos que se verifican en este tipo de trabajo.

Las funciones encomendadas a los servidores policiales están estrechamente relacionadas con potenciales factores de estrés físicos y psicológicos de la misma institución y fuera de ella, que pueden verse agravados por la naturaleza del trabajo. En este sentido, Maslach (2009b): señala como labores de "alto riesgo" y que podrían presentar cuadros de 
estrés: los docentes, enfermeros, trabajadores sociales, médicos y policía. Caba destacar que para Di Martino y Casanueva (1994): Ios agentes de policía ocupan el segundo lugar en presentar síntomas de estrés laboral, superados solamente por los trabajos de minería

Considerando a Durán (2001): los riesgos psicosociales y el burnout afectan directamente a la esfera emocional y afectiva, generando secuelas en su comportamiento dentro del entorno individual, familiar, social y laboral. Acorde con esto, la Organización Internacional del Trabajo (2016): presenta que los trabajadores de servicios públicos que atienden urgencias experimentan un estrés considerable relacionado con el trabajo, los servidores públicos se ven afectados a nivel psicológico por acontecimientos críticos relacionados con sus actividades diarias.

La importancia de esta investigación se basa en identificar los riesgos psicosociales y burnout que mantiene esta población, debido a la complejidad de sus funciones, pues el contacto directo de esta población con la ciudadanía y la apresurada demanda de soluciones en el menor tiempo posible, generando ciertas condiciones adversas en el cumplimiento de sus funciones designadas.

El aporte clínico se direcciona tanto a la Institución Policial como a la sociedad ecuatoriana en general, identificando los factores de riesgo generados en el trabajo diario, del personal que cumple funciones operativas y administrativo de la DINASED en Quito, Ecuador, debido a la cronicidad de sus funciones en las Unidades tales como: Muertes Violentas, Desaparecidos, antisecuestros y extorsión, unidad de análisis de la información entre otros.

El proyecto contribuye al finalizar esta investigación a identificar las condiciones mentales de la población en estudio, mediante los instrumentos aplicados, con cada uno de los factores generados por los riesgos psicosociales como el burnout en la DINASED. Mediante la presentación de resultados, se pretende visualizar y sensibilizar a los diversos estamentos de 
control, sobre el estado psicológico del personal directivo y técnico operativo que labora en la DINASED y el comprometimiento del Director Nacional de esta dirección, en relación con el cumplimiento de las recomendaciones realizadas en cuanto a intervención de riesgos psicosociales y burnout.

El papel del psicólogo es el de velar por el bienestar de cada individuo con la finalidad de potenciar su calidad de vida. La intervención psicológica pretende evitar carencias y patologías en la salud mental del usuario tomando un papel protagonista e independiente, transformándose en un funcionario activo de cambio tanto en la cotidianidad como en condiciones de crisis, con la finalidad de potencializar sus habilidades y competencias.

En atención a Eguzquiza (2013): el beneficio de lograr las metas trazadas requiere de un abordaje y colaboración multidisciplinario, en el cual la labor del psicólogo se articula con el de cada miembro de la organización. Conforme a Camacho (2019): entre las principales competencias del profesional en salud mental que tome a cargo este proyecto, está el de evaluar las particularidades de los individuos, grupos, estructuras y situaciones utilizando procesos apropiados, en cada uno de los contextos apreciables, para las actividades solicitadas mediante la evaluación individual, grupal, organizacional y situacional.

Otras recomendaciones que se extienden, para la revisión bibliográfica realizada, apunta a la consideración de los siguientes aspectos:

- Intervenir psicológicamente mediante la identificación, preparación y realización de intervenciones apropiadas para conseguir los objetivos propuestos.

- Proporcionar una constante comunicación con el/los usuarios que permita satisfacer las necesidades y expectativas de estos.

- Elaborar informes clínicos detallados que permitan brindar información sobre la atención realizada. 
Esta investigación tiene como objetivo identificar el riesgo psicosocial y burnout en el personal directivo y técnico operativo de la Dirección Nacional de Delitos Contra la Vida, Muertes Violentas, Desapariciones, Extorsión y Secuestro de la Policía Nacional (DINASED), de manera que sea posible constatar el impacto tanto a nivel físico como emocional al que se encuentran expuestos estos servidores públicos, por encontrarse en contacto permanente con casos de desaparición de personas, muertes violentas, tentativas de asesinato y secuestro.

\section{Metodología}

La presente investigación se realiza mediante un enfoque cuantitativo, determinando porcentualmente el riesgo psicosocial y burnout tanto en el personal directivo como en el técnico operativo, de tipo experimental ya que se controla las variables para delimitar la relación entre ellas y con un corte trasversal, ya que la presente investigación es realizada en un tiempo y espacio determinado.

El análisis se realiza en una población de 170 servidores policiales de nivel Directivo y Técnico Operativo de la Dirección Nacional de Delitos Contra la Vida, Muertes Violentas, Desapariciones, Extorsión y Secuestro de la Policía Nacional (DINASED). Los participantes cumplen con los siguientes criterios de inclusión: son servidores policiales activos, corresponden al personal que pertenece a la DINASED, son hombres y mujeres de Nivel Directivo y Técnico Operativo de esta Dirección. Quedan excluidos del estudio los servidores policiales que no pertenezcan a esta dirección.

El análisis se realiza a través de la Aplicación de Reactivos Psicométricos tales el cuestionario de riesgos psicosociales $3.1 \mathrm{y}$ el Cuestionario de Maslach Burnout Inventory, herramientas que fueron seleccionadas debido a su fundamento científico, diseño, fiabilidad y validez.

El Cuestionario de Riesgos Psicosociales FPSICO 3.1, obtiene 
indicadores tales como: Tiempo de Trabajo, Autonomía, Carga de trabajo, Demandas Psicológicas, Variedad y Contenido del Trabajo, Participación y Supervisión, Interés por el Trabajador y Compensación, Desempeño del Rol, Relaciones y Apoyo Social. Tomando como rango muy elevado, elevado, riesgo moderado, situación adecuada.

El Cuestionario de Maslach Burnout Inventory, mide 3 factores esenciales: Cansancio Emocional, Despersonalización y Realización Personal, considerando los rangos de: alto, medio y bajo.

Este estudio se lo ejecuta en 4 fases, inicialmente con un proceso de socialización relacionado a los posibles riesgos que generan los factores psicosociales dentro del ámbito laboral, Toma de reactivos, Análisis de Resultados Obtenidos, valoración y comparación de los resultados.

\section{Resultados}

De acuerdo con la información obtenida de la aplicación de los instrumentos, se procedió al análisis e interpretación de los resultados, enfocándose en determinar la existencia o ausencia del riesgo psicosocial y niveles de burnout dentro de la DINASED en Quito, Ecuador.

Gráfico 1. Factores de Riesgo Psicosocial en el Personal de Servidores Policiales que cumplen Funciones Operativas priorizando en nivel de Riesgo Muy Elevado y Elevado.

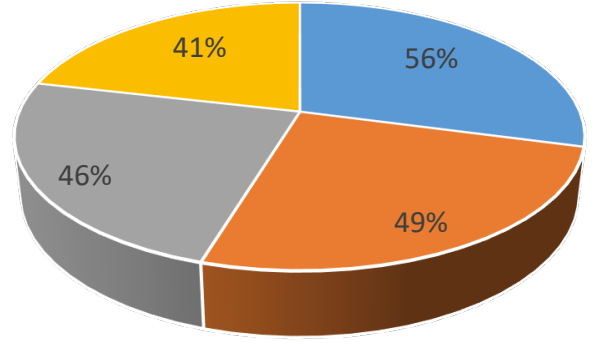

- Tiempo de Trabajo

- Demandas Psicológicas

- Participación y Supervisión

- Carga de trabajo

Fuente: Los Autores (2020). 
En el gráfico 1, se puede apreciar que el "Tiempo de Trabajo", obtuvo el $56 \%$ el mismo que estuvo relacionado con trabajar sábados, domingos, días feriados tiempos de descanso a la semana, determinando el impacto del tiempo del trabajo en relación a la vida personal, familiar y social, tomando en cuenta que los miembros de la Institución Policial brindan sus servicios las 24 horas del día, los 365 días al año. En el caso de los servidores policiales es parte de su misión y vocación de servicio. En cuanto al personal de servidores policiales que cumplen funciones Operativas, se evidenció una puntuación dentro de la escala valorativa de "Muy elevado" y "Elevado" en diversos aspectos.

"Demandas Psicológicas" presentó un resultado del $49 \%$ el mismo que se refiere a la naturaleza de las exigencias en las cuales se consideran dos factores principales estas pueden ser cognitivas (información, memorización, razonamiento, solución de problemas) y de tipo emocionales (reprimir sentimientos y compostura) en el caso de este grupo de servidores policiales se requiere del desarrollo de destrezas y habilidades dentro del campo investigativo.

Por lo que el nivel de estrés fue alto ya que trabaja directamente con seres humanos, y esto generó frustraciones frecuentes ya que este personal identifica la angustia del usuario y sus familiares, sintiendo en algún momento impotencia en relación a las perspectivas del proceso investigativo y resolución del caso, está expuesto a situaciones como la cooperación de los familiares y la resolución de sus conflictos, temor a traslados laborales fuera de su entorno familiar, estructura organizacional tensa debido al tiempo de resolución que se determina para cada caso, evidenciando sentimientos de frustración, desilusión, impotencia, irritabilidad, inhibición, indiferencia, desgaste emocional o desequilibrio entre su vida personal, familiar, laboral y social.

"Participación y Supervisión" se obtiene una puntuación del $46 \%$ el mismo que está relacionado con el impacto del trabajador a través de su 
participación en diferentes aspectos del trabajo y la segunda que ejerce la organización sobre el trabajador mediante la supervisión de sus actividades, la falta o a su vez el incorrecto funcionamiento de herramientas. En este caso los servidores policiales operativos participan en actividades de riesgo, condiciones ambientales adversas, bajo presión, sujetos a un tiempo determinado de resolución de casos, generando estados de estrés al no cumplir con el objetivo y no presentar resultados los mismos que son requeridos por su jefe inmediato.

"Carga de trabajo" arrojó un resultado del 41\% constituye el nivel de demanda de trabajo al que el trabajador hizo frente, considerando los siguientes aspectos, presión de tiempo, esfuerzo de atención, cantidad y dificultad de la tarea, en el caso de este grupo de servidores policiales podemos analizar que la demanda supera al número de servidores policiales frente a los casos a resolver.

El segundo grupo que fue evaluado con este instrumento corresponde al personal de servidores policiales que cumplen funciones administrativas, los resultados que arrojó este grupo se muestran en la siguiente gráfica:

Gráfico 2. Factores de Riesgo Psicosocial en el Personal de Servidores Policiales que cumplen Funciones Administrativas priorizando en nivel de Riesgo Muy Elevado.

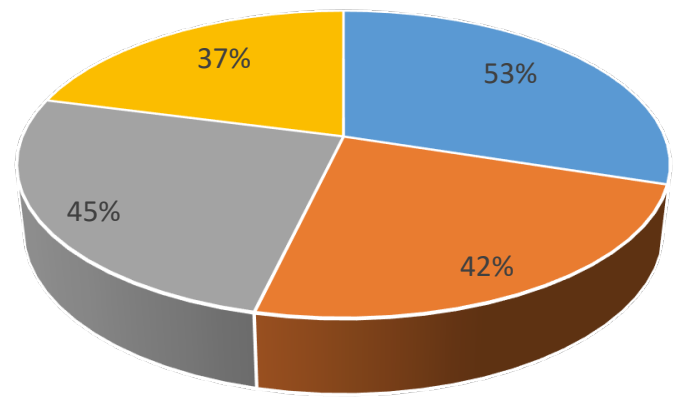

- Tiempo de trabajo

- Demanda Psicológica

- Participación y Supervisión

- Carga de Trabajo

Fuente: Los Autores (2020). 
Para el gráfico 2, se presenta que el "Tiempo de trabajo", puntuó 53\% en las funciones administrativas este factor guardó similitud con la función operativa, que también laboraron los sábados, domingos, y días feriados ya que el trabajo del personal de apoyo y análisis de la información constituyeron un ente fundamental para la ejecución del trabajo operativo.

"Demanda Psicológica" obtuvo el $42 \%$ dentro del desempeño de funciones administrativas se requiere de alta exigencia cognitiva para el análisis de la información debiendo desarrollar habilidades y destrezas las mismas que están vinculadas con seleccionar información importante, memorizar, con el propósito de establecer estrategias las mismas que son trasmitidas al personal operativo.

"Participación y Supervisión" presentó un 45\% suponiendo que los servidores policiales estuviesen sujetos a trabajos bajo presión, teniendo que establecer estrategias de acción inmediata las mismas que fueron reportadas al jefe del caso, el cual supervisa que estas estrategias sean trasmitidas al personal operativo dentro del tiempo establecido.

"Carga de Trabajo" equivale al $37 \%$ sus funciones exigen esfuerzo de atención y capacidad resolutiva inmediata teniendo como limitantes un análisis más exhaustivo de la información, exacerbando estados de desgaste físico y mental.

Dentro de este estudio también pudimos evidenciar que esta Dirección cuenta con factores que dentro de la escala valorativa presentan una situación adecuada, la misma que deberá ser fortalecida ya que constituye un aspecto positivo dentro del ámbito laboral.

Se pueden destacar factores adecuados dentro del desempeño de funciones operativas, tales como la variedad del contenido del trabajo, autonomía, interés por el trabajo y compensación, relación y apoyo social, desempeño del rol, situación administrativa adecuada.

A continuación, presentamos los resultados obtenidos en la aplicación 
del Cuestionario de Maslach Burnout Inventory. Este fue aplicado a la misma población de servidores policiales que formaron parte de este estudio; con la variable de que los servidores policiales tenían que colocar su nombre y apellido, con el propósito de mantener un registro del personal evaluado y se pueda tomar en cuenta a los servidores policiales que requieran de intervención psicológica individual, los resultados que arrojó este cuestionario se observan en las gráficas a continuación.

En este primer factor el $84 \%$ del personal se encontró dentro del nivel bajo, el $10 \%$ en el nivel medio y el $6 \%$ en el nivel alto, dentro de esto debemos mencionar que el departamento de muertes violentas reportó un $5 \%$ siendo este el indicador más alto en comparación con los demás departamentos, dentro del análisis de este resultado pudimos identificar que este grupo de servidores policiales realizaron actividades como de levantamiento de cadáveres e investigación de muertes violentas, le sigue con el $4 \%$ el personal de dirección, dentro del cual se encuentran quienes trabajaron en la dirección administrativa, secretaria, logística y talento humano, este porcentaje se debe a que el personal ha sido rotado como política interna de esta dirección debido a la permanencia de mucho tiempo en la función operativa, los departamentos de Unidad Antisecuestro y extorsión y la Unidad de Personas Desaparecidas, tienen un $1 \%$ cerrando con un porcentaje de $0 \%$ el Departamento de Análisis de Información.

El factor despersonalización, arrojó un indicador del $67 \%$ el nivel bajo, $18 \%$ el nivel medio y $15 \%$ el nivel alto, en este indicador el personal de muertes violentas puntúo $11 \%$ validando el resultado con el factor anterior el mismo que se relaciona con cansancio emocional debido a la complejidad de sus actividades y a exposición del dolor e impacto emocional al momento de cumplir con sus funciones, el personal de dirección $6 \%$, Unidad Antisecuestro y extorsión y la Unidad de Desaparición obtuvieron un 4\%, que equivale al desempeño de su actividades generando un impacto global y el $1 \%$ 
corresponde al personal que labora en el departamento de Análisis de la Información. En función de dicha escala, los factores mencionados son indicadores de burnout, por lo que en conclusiones se analizará este fenómeno en este grupo de trabajo.

Los resultados en el indicador realización personal, continuaron la secuencia anterior, presentando en el indicador alto un $65 \%$, medio un $21 \%$ y bajo $14 \%$, para confirmar el resultado de burnout, este indicador debería presentar un indicador alto en el porcentaje bajo, sin embargo, esto no ocurre en este grupo de personas. En los resultados por Unidades, tenemos que el personal de Muertes Violentas obtuvo un 11\%, siendo este el mayor indicador dentro del grupo bajo, el grupo de dirección el 6\%, la Unidad de Personas desaparecidas un $4 \%$, el personal de la UNASE obtuvo un $2 \%$, finalizando con el Departamento de Análisis de Información con el 1\%, como los resultados más bajos.

\section{Conclusiones}

La herramienta de valoración FPSICO 3.1 y el Cuestionario de Maslach Burnout Inventory, esto permite identificar las condiciones emocionales de los servidores policiales de nivel directivo y técnico operativo de la Dirección Nacional de Delitos Contra la Vida, Muertes Violentas, Desapariciones, Extorsión y Secuestro de la Policía Nacional (DINASED) en Quito, Ecuador, evidenciando de esta manera que esta Dirección presenta factores de riesgo en una escala valorativa de muy elevado, elevado en 4 factores principales: Tiempo de trabajo, Demanda Psicológica, Participación-Supervisión y Carga de Trabajo. Dichos factores están relacionados con el deterioro de las condiciones físicas y emocionales en mayor o menor intensidad, debido a la unidad perteneciente y a las funciones a realizar en su ámbito laboral.

Por otro lado, mediante el cuestionario de Burnout se identifica a través de una escala valorativa de alto, medio y bajo, factores tales como: cansancio 
emocional, despersonalización y realización personal; identificando que en la unidad de Muertes Violentas (MV) prevalece una alta puntuación en cuanto al mantenimiento de cansancio emocional y despersonalización resultados que presumimos se debe a la función que desempeñaron.

Dentro de las funciones y actividades que realiza esta Unidad tenemos: Levantamientos de cadáveres por (muerte natural, accidental y muerte violenta) procedimientos en tentativas de asesinatos, levantamiento de indicios en el lugar de los hechos, fijación, levantamiento y etiquetado de indicios, en casos de muertes violentas examen visual externo del cuerpo, toma de versiones a personas involucradas dentro del hecho, observación de la escena en primera instancia así como también la posición del cuerpo (cadáver) y procesos de descomposición dentro de la escena.

También se puede identificar dentro de la escala valorativa que existen algunos factores que puntuaron el mantenimiento de una situación laboral adecuada, entre ellos el más significativo es Variedad y contenido, y en cuanto al cuestionario de Maslach prevaleció la Realización Personal en la Unidad de Personas desaparecidas.

Finalmente, los riesgos psicosociales y burnout afectaron de forma significativa en la esfera bio psico social y laboral de individuo impactando su vida y su interacción con el medio, por lo que este estudio identificó dichos riesgos con la finalidad de generar cambios que permitan un mejor desempeño en todas las esferas del servidor policial.

\section{Referencias}

Álvarez, F. (2006). Salud Ocupacional. Bogotá, Colombia: Ecoe Ediciones. Camacho, C. (2019). Una mirada desde el Síndrome de Burnout hacia la Gerencia Integral en el Siglo XXI. Revista Scientific, 4(13), 40-59, eISSN: 2542-2987. Recuperado de:

https://doi.org/10.29394/Scientific.issn.2542-2987.2019.4.13.2.40-59 
Durán, M. (2001). El Síndrome de Burnout en organizaciones policiales: Una aproximación secuencial. Tesis Doctoral. Málaga, España: Facultad de Psicología de la Universidad de Málaga. Recuperado de: http://www.biblioteca.uma.es/bbldoc/tesisuma/16272675.pdf

Di Martino, V., \& Casanueva, B. (1994). Por la prevención del estrés en el trabajo: la estrategia de la OIT. Salud y trabajo, (102), 17-23, ISSN: 0210-6612. España: Instituto Nacional de Seguridad de Higiene en el Trabajo.

Eguzquiza, D. (2013). Roles y funciones del psicólogo de la intervención social. Madrid, España: Consejo General de Colegios Oficiales de Psicólogos. Recuperado de:

http://www.copao.com/index.php/publicaciones/doc view/160-roles-yfunciones-del-psicologo-de-intervencion-social

Maslach, C. (2009). Comprendiendo el Burnout. Ciencia \& Trabajo, 11(32), 37-43, ISSN: 0718-2449. Chile: Fundación Científica y Tecnológica ACHS.

Moreno, B. (2011). Factores y riesgos laborales psicosociales: conceptualización, historia y cambios actuales. Medicina y Seguridad del Trabajo, 57(Supl. 1). 4-19, e-ISSN: 1989-7790. Recuperado de: http://dx.doi.org/10.4321/S0465-546X2011000500002 MDT (2017). Normativa erradicación de la discriminación en el ámbito laboral. Acuerdo Ministerial No. MDT-2017-0082. Quito, Ecuador: Ministerio del Trabajo.

Organización Internacional del Trabajo (2016). Estrés en el trabajo: Un reto colectivo. Primera Edición, ISBN: 978-92-2-330641-0. Turín, Italia: Centro Internacional de Formación de la OIT. 


\section{Nathalie Soledad López Arroyo}

e-mail: nslopez.mpp@uisek.edu.ec

Nacida en Quito, Ecuador, el 23 de septiembre del año

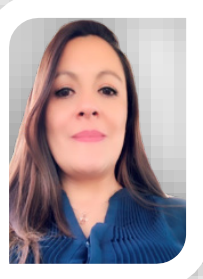
1975. Doctora en Psicología Clínica por la Universidad Central del Ecuador (UCE); Magíster en Desarrollo del Talento Humano por la UCE; y Magíster en Psicoterapia por la Universidad Internacional SEK (UISEK); Tratante del Servicio de Salud Mental de la Policía Nacional del Ecuador, Hospital Quito N. ${ }^{\circ}$ 1; Docente universitaria en temas de criminología. 
OAI-PMH: http://www.indteca.com/ojs/index.php/Revista_Scientific/oai

Artículo Original / Original Article

\section{Erika Elizabeth Torres Rueda}

e-mail: eetorres.mpp@uisek.edu.ec

Nacida en Quito, Ecuador, el 19 agosto del año 1984.

Psicóloga clínica; Magíster en Psicología con mención en Psicoterapia por la Universidad Internacional SEK (UISEK); Analista en la Alcaldía del Distrito Metropolitano de la ciudad de Quito. 


\section{Carmen Eugenia Mora Mora}

e-mail: cemora.mpp@uisek.edu.ec

Nacida en Quito, Ecuador, el 3 de diciembre del año 1976.

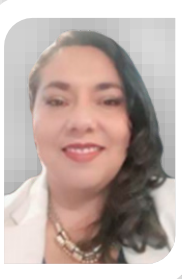

Licenciada en Ciencias Sociales Políticas y Económicas por

la Universidad Técnica Particular de Loja (UTPL); Psicóloga

Clínica por la Universidad Central del Ecuador (UCE); y

Magister en Psicología Mención Psicoterapia por la

Universidad Internacional SEK (UISEK); Docente en

instituciones de nivel medio de la ciudad de Quito; En atención particular, he participado en campañas de prevención de adicciones, psicoterapia individual, pareja, grupal, y familiar con distintos grupos etarios. 
OAI-PMH: http://www.indteca.com/ojs/index.php/Revista_Scientific/oai

Artículo Original / Original Article

\section{Esteban Ricaurte Ricaurte} e-mail: esteban.ricaurte@uisek.edu.ec

Nacido en Quito, Ecuador, el 7 de junio del año 1977.

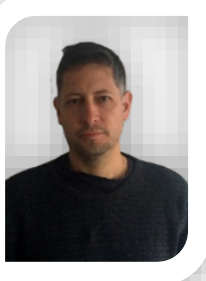
Psicólogo clínico; con maestría en psicoterapia cognitiva; socio fundador del centro de psicoterapia Aysana; Fundador y vicepresidente de la Asociación de Psicoterapia cognitiva del Ecuador (APCE); cuento con 10 años de experiencia docente; profesor titular de la Universidad Internacional SEK (UISEK), en pregrado y maestría; con especialidad en adicciones. 